\title{
A refractory case of CDK4-amplified spinal astrocytoma achieving complete response upon treatment with a Palbociclib-based regimen:a case report
}

\author{
Jietao Lin ${ }^{1,2 \dagger}$, Ling $\mathrm{Yu}^{1,2+}$, Yuanfeng $\mathrm{Fu}^{3}$, Hanrui Chen ${ }^{1,2}$, Xinting Zheng ${ }^{1,2}$, Shutang Wang ${ }^{1,2}$, Chan Gao ,
} Yang CaO ${ }^{1,2+}$ and Lizhu Lin ${ }^{1,2^{*}+}$ (D)

\begin{abstract}
Background: Spinal cord astrocytoma is a rare neoplasm, and patients usually recur within months after surgery. There is currently a lack of consensus regarding post-operative treatment. Clinical data on the activity of systemic treatment like chemoradiotherapy and anti-angiogenic agents also remained scant. Next-generation sequencing (NGS) -based genomic profiling thus may help identify potential treatment options for a subset of patients that harbor actionable genetic alterations.

Case presentation: We reported for the first time a refractory case of grade III spinal cord astrocytoma that underwent two surgeries but eventually progressed following post-operative chemoradiotherapy plus bevacizumab. Hybridization capture-based NGS using a 381-gene panel disclosed cyclin dependent kinase 4 (CDK4) amplification and after receiving a triplet regimen containg palbociclib for 15 months, the patient achieved complete response.

Conclusions: This case demonstrated the importance of genetic profiling and the benefit of a multi-modality treatment strategy in cancer management.
\end{abstract}

Keywords: Spinal astrocytoma, next-generation sequencing, Palbociclib, Targeted therapy

\section{Background}

Astrocytomas are a rare group of glial neoplasms of the central nervous system (CNS). They arise from astrocytes, supporting cells of the nervous system, and only $3 \%$ of astrocytomas are found in the spinal cord [1]. Spinal cord astrocytoma (SCA) comprises $2.1 \%$ of all adult primary spinal cord tumors, which in turn,

\footnotetext{
* Correspondence: lizhulin26@yahoo.com

†jietao Lin and Ling Yu contributed equally to this work. Yang Cao and Lizhu Lin contributed equally to this work.

'Oncology Center, the First Affiliated Hospital of Guangzhou University of Chinese Medicine, 16th Airport Road, Guangzhou 510405, Guangdong, China ${ }^{2}$ Guangzhou University of Chinese Medicine, 12th Airport Road, Guangzhou 510405, Guangdong, China

Full list of author information is available at the end of the article
}

accounts for $2-4 \%$ of all CNS tumors [2, 3]. The prognosis of SCA patients depends on the tumor grade (grade I-IV according to World Health Organization criteria) and duration of symptoms before diagnosis, where high-grade ones are usually highly aggressive and may cause neurological deficiency or even death [4]. There are currently limited treatment options available for SCAs. Surgery serves as the initial treatment modality; however, complete resection is often not possible due to the infiltrative nature of astrocytoma [1, 4]. Although post-operative spinal radiation has been adopted worldwide to prevent recurrence, its exact role in SCA management remained controversial because low-grade SCAs may benefit minimally from radiotherapy due to

(c) The Author(s). 2020 Open Access This article is licensed under a Creative Commons Attribution 4.0 International License, which permits use, sharing, adaptation, distribution and reproduction in any medium or format, as long as you give appropriate credit to the original author(s) and the source, provide a link to the Creative Commons licence, and indicate if changes were made. The images or other third party material in this article are included in the article's Creative Commons licence, unless indicated otherwise in a credit line to the material. If material is not included in the article's Creative Commons licence and your intended use is not permitted by statutory regulation or exceeds the permitted use, you will need to obtain permission directly from the copyright holder. To view a copy of this licence, visit http://creativecommons.org/licenses/by/4.0/. The Creative Commons Public Domain Dedication waiver (http://creativecommons.org/publicdomain/zero/1.0/) applies to the data made available in this article, unless otherwise stated in a credit line to the data. 
low spontaneous recurrence rates while high-grade SCAs generally have low sensitivity to radiation $[1,4]$. Likewise, established chemotherapy regimens such as temozolomide, administered alone or in combination with bevacizumab, are also considered to have limited value in treating SCAs since they have not been systematically examined or validated in large prospective studies [4]. Multi-modality therapy is, therefore, of paramount importance in such a scenario and next-generation sequencing (NGS)-guided targeted therapy may serve as a last resort for certain patients. We herein reported a CDK4amplified case of SCA achieving complete response following multi-modality therapy containing palbociclib.

\section{Case presentation}

A 38-year-old man with a decade's history of chronic hepatitis B virus infection presented with lower back pain in March, 2016. He did not have any hereditary diseases, a family history of cancer, a history of trauma, or any other chronic medical conditions. Spinal magnetic resonance imaging (MRI) disclosed a mass measuring $10 \times 14 \mathrm{~mm}$ in the 10th thoracic segment of his spinal cord on March 31st. The tumor was surgically removed on April 13th, 2016. Post-surgical pathology revealed anaplastic astrocytoma (WHO grade III). Immunohistochemical staining demonstrated that the tumor was positive for Vimentin (+++), GFAP (+), S-100 (+), Syn (focally +), Ki-67(70\% +), and p53 (partly + ), but negative for CgA and EMA (Fig. 1, Supplemental Table 1). An Olympus BX41 microscope with a 10× ocular lens and a 20x objective lens was used for microscopy and an MShot MD3 microscope camera along with Mshot Image Analysis System was used for image acquisition. The images were acquired at a resolution of $96 \mathrm{dpi}$ and
Adobe Photoshop was used to enhance the resolution of the images to 300 dpi. Both H3.3 histone A (H3F3A) and histone cluster 1, H3b (HIST1H3B), which are commonly mutated in pediatric midline glioma and sometimes in adult patients, were shown to be wild-type using fluorescence in situ hybridization (FISH). The patient did not harbor any dehydrogenase $(I D H)$ mutations or $1 \mathrm{p} / 19 \mathrm{q}$ co-deletion, either according to FISH.

On September 18th, 2016, a follow-up MRI scan revealed local recurrence of the primary lesion, and a second surgical excision was performed on September 26th, 2016. Histological examination confirmed the initial pathological diagnosis of anaplastic astrocytoma. Following surgery, adjuvant chemotherapy consisting of 4 cycles of nedaplatin (50 mg ivgtt D1-D3) and temozolomide (250 mg po D1-D5) was administered every 28 days. In March, 2017, the patient experienced an onset of progressive numbness and weakness in the lower limbs. The dysesthesias and weakness in the lower limbs became intensified later in April. Positron emission tomography-computed tomography (PET-CT) showed a hypermetabolic lesion in the 10th thoracic spinal cordon April 17th, 2017 (Fig. 2.A.). From April 24th, 2017 to April 29th, 2017, the patient underwent gamma knife radiosurgery at a marginal dose of $40 \mathrm{~Gy}$ and this was followed by four cycles of chemotherapy comprising bevacizumab $(500 \mathrm{mg}$ ivgtt D1), irinotecan $(190 \mathrm{mg}$ ivgtt D1) and temozolomide $(250 \mathrm{mg}$ po D1-D5) administered every 28 days.

The adjuvant chemoradiotherapy failed to control disease progression as a CT scan conducted on July 20th, 2017 indicated a second recurrence. Resected tissue sample obtained during the second surgery was therefore subjected to NGS analysis using a 381-gene panel (3DMedicine Clinical Laboratory, China) (Supplemental
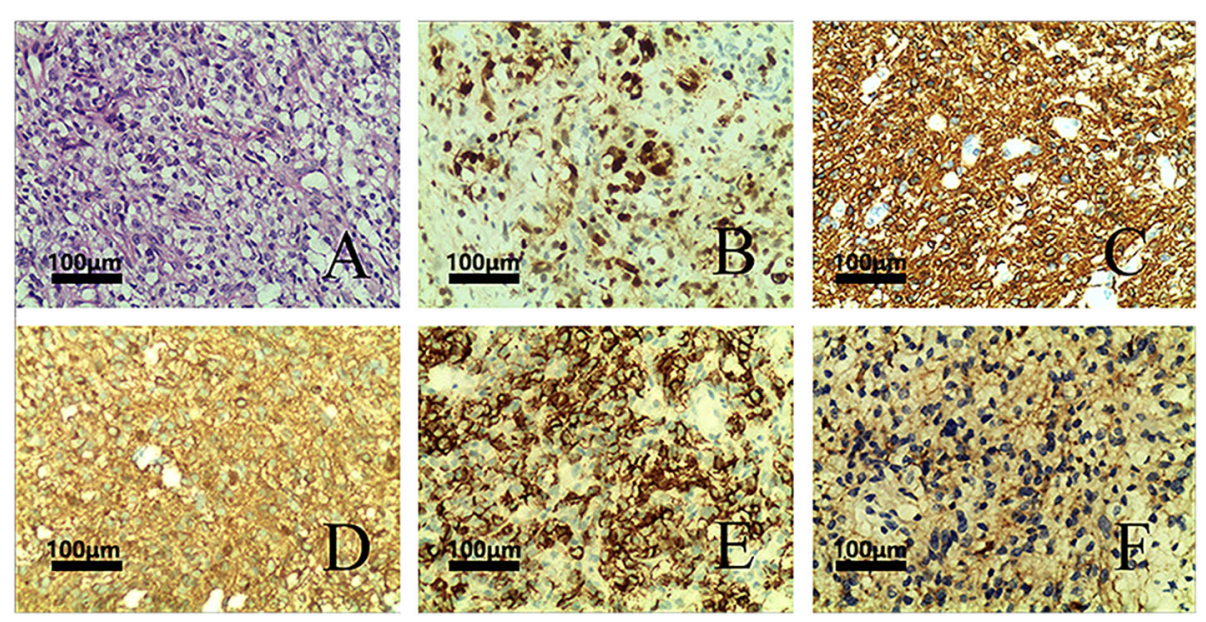

Fig. 1 Histologic features of the tumor. a H\&E section showing diffuse invasion of tumor cells with abundant cytoplasm, indicative of anaplastic oligodendrocytic astrocytoma, WHO III grade; b) IHC showing KI-67: 70\% (+); c) IHC showing focal staining of GFAP focal; (+) d) IHC showing strong diffuse staining of Vimentin (+); e) $I H C$ showing CD56 partly (+); f) $I H C$ showing Syn partly (+). Original magnifications: a-f:200X. H\&E:haemotoxylin and eosin. IHC: immunohistochemistry 

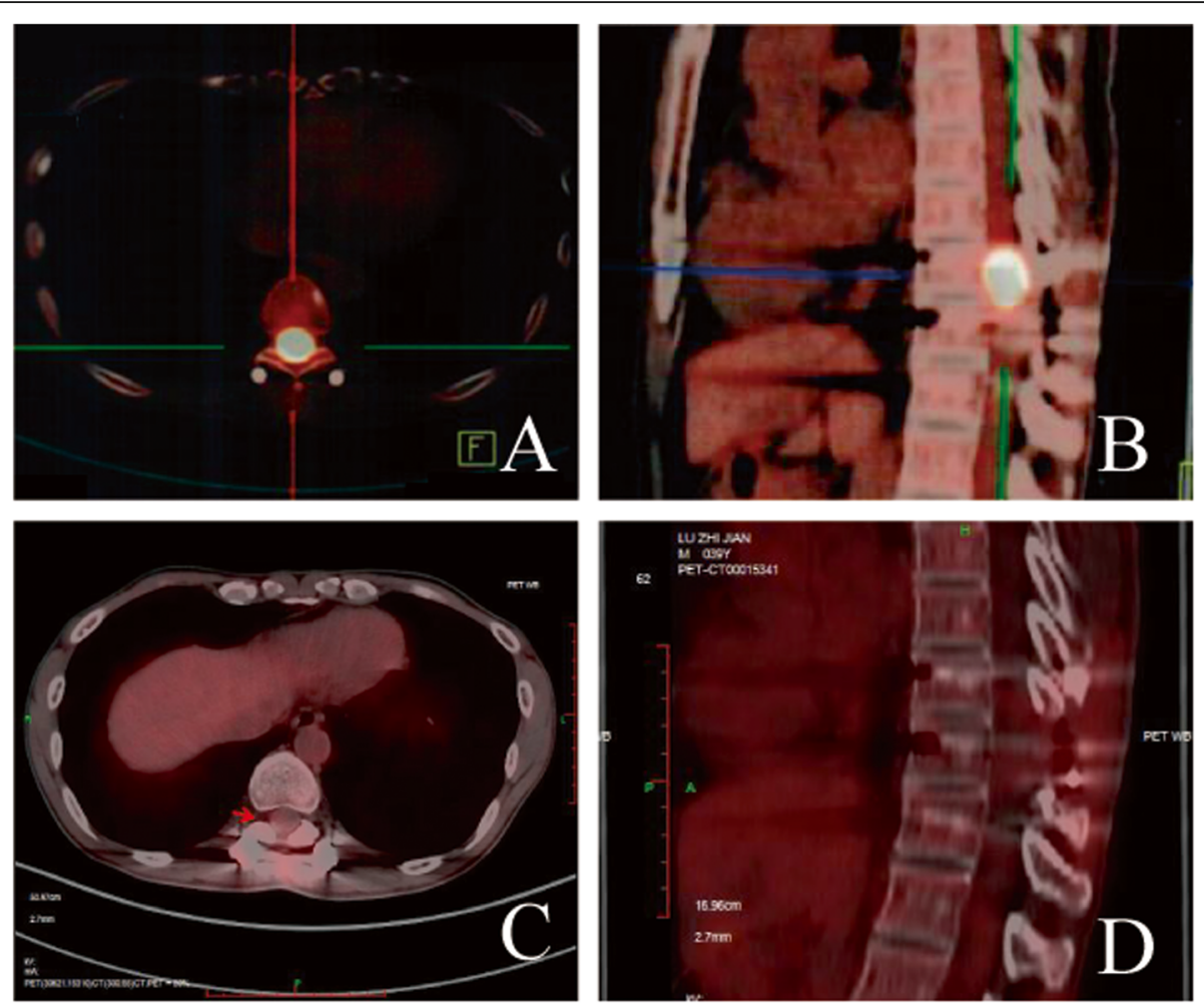

Fig. 2 Positron emission tomography-computed tomography (PET-CT) scans showing the second recurrence in the 10th thoracic spinal cord on April 17th, 2017 (a and b), and complete response on August 24th, 2018 (c and d)

Table 2). As summarized in Table 1, genetic alterations identified included amplification of the genes encoding cyclin dependent kinase 4 (CDK4), murine double minute-2 (MDM2), fibroblast growth factor receptor substrate 2 (FRS2), and GLI family zinc finger 1 (GLI1) and point mutations in WEE1 G2 checkpoint kinase (WEE1, c. 1385-1 G > A) and protein tyrosine phosphatase non-receptor type 11 (PTPN11, p.E69K). The patient was also found to be microsatellite stable (MSS) and hence was not likely to benefit from immunotherapy. Taken together, among all the genetic abberations identified, CDK4 amplifcation was the only one that was

Table 1 Gene mutational profile of the spinal astrocytoma patient by next-generation sequencing

\begin{tabular}{lll}
\hline & mutation & Mutation abundance (\%)/copy number \\
\hline CDK4 & amplification & 32 \\
WEE1 & c.1385-1G > A & $38.30 \%$ \\
MDM2 & amplification & 37 \\
PTPN11 & p.E69K & $31.30 \%$ \\
FRS2 & amplification & 37 \\
GLI1 & amplification & 32
\end{tabular}

Abbreviations: CDK4 = cyclin dependent kinase 4, WEE1 = WEE1 G2 checkpoint kinase, $\mathrm{MDM} 2=$ MDM2 proto-oncogene, PTPN11 = protein tyrosine phosphatase non-receptor type $11, \mathrm{FRS} 2=$ fibroblast growth factor receptor substrate $2, \mathrm{GLI} 1=\mathrm{GLI}$ family zinc finger 1 potentially targetable. Amplification of $C D K 4$ may result in dysregulation of the cycline-D- cyclin-dependent kinase $4 / 6$ (CDK4/6)-INK4-Rb pathway and eventually cause cell cycle progression and tumorigenesis [5]. Palbociclib, a selective oral inhibitor of $C D K 4 / C D K 6$, binds to the ATP pockets of CDK4/6 and leads to cell cycle arrest at G1 phase [6]. Although palbociclib had not been approved for treating CNS malignancies, in a phase II study conducted on 30 patients diagnosed with CDK4amplified advanced well-differentiated or dedifferentiated liposarcoma (WD/DDLS), palbociclib generated an estimated 12-week progression free survival (PFS) rate of $66 \%$, well exceeding the pre-specified $40 \% 3$ month PFS rate to consider the study positive [7]. The patient was, therefore, started on four cycles of palbociclib (125 mg po d1-21 q4w) plus temozolomide (250 mg po d1-5 q4w) on September 10th, 2017. MRI scans conducted every 2 months showed continuous tumor regression, and the symptoms also became stable with the sensation in the lower limbs gradually alleviated. Temozolomide was discontinued on March 28th, 2018 due to intolerable myelosuppression while palbociclib was continued for another two months before temozolomide was resumed along with apatinib when the patient's conditions improved. Apatinib, in combination with chemotherapy, has been shown to be both effective and 
tolerable in adult patients with recurrent glioma and was hence included in the treatment regimen [8]. The triplet regimen lasted for two months, and a PET-CT scan conducted on August 24th, 2018 showed complete response (Fig. 2.B.). Apatinib was discontinued on September 15th, 2018, and the patient stopped taking temozolomide and palbociclib on April 20th, 2019. The patient was alive till the last follow-up on August 15th, 2019.

\section{Discussion and conclusions}

Spinal astrocytomas are rare intramedullary CNS tumors, and evidence regarding efficacious systemic therapeutic agents is too scant to inform specific recommendations according to the National Comprehensive Cancer Network (NCCN) guidelines for central nervous system cancers [9]. We herein reported a case of spinal astrocytoma, where the patient underwent two surgeries and recurred three times. Adjuvant doublet chemotherapy following the first resection and chemoradiotherapy after the second excision both failed to thwart disease progression. A regimen containing palbociclib was therefore adopted upon identification of CDK4 amplification using NGS-based genetic testing. The patient responded well and achieved complete response after 11 months of treatment.

This patient was indeed a rare case because he was triple-negative for IDH mutations, TERT promoter mutations and $1 \mathrm{p} / 19 \mathrm{q}$ co-deletion, which is observed in only $7 \%$ of spinal astrocytoma patients according to a previous report, Wild-type IDH1 or IDH2 is associated with an increased risk of aggressive disease, and prognosis for triple-negative patients are even worse [10]. This is consistent with the fact that the patient in this case recurred in five months after the first surgery.

The cyclin D (CCND1)-CDK4/6-INK4-Rb pathway is a key regulator of the G1-S transition in the cell cycle. When activated by mitogenic signaling, CCND1 binds with $C D K 4 / 6$ to form a complex which phosphorylates $\mathrm{Rb}$ and thereby releases E2F from the transcriptionally repressive $\mathrm{Rb}-\mathrm{E} 2 \mathrm{~F}$ complex. E2F is thus free to promote transcription of genes required for cell cycle progression and DNA replication [5]. Amplification of the CCND1, $C D K 4$, or $C D K 6$ genes or loss-of-function mutations in cyclin-dependent kinase inhibitor 2A (CDKN2A) are the primary mechanisms for overactivation of the CCND1CDK4/6-INK4-Rb pathway [6]. It was previously reported that CDK4 amplification occurs in $15 \%$ of malignant gliomas [10]. Palbociclib is the first-in-class CDK4/ 6 inhibitor and has been granted FDA approval for either first-line use in combination with an aromatase inhibitor (AI) in hormone receptor positive $(\mathrm{HR}+)$ human epidermal growth factor receptor 2 negative (HER2-) metastatic breast cancer (MBC) or in pretreated $\mathrm{MBC}$ patients in combination with fulvestran. Although it has not been approved yet to treat CDK4-altered solid tumors, palbociclib directly targets $C D K 4$ by binding to its ATP pocket. Moreover, it was previously shown that palbociclib monotherapy produced a favorable PFS rate in liposarcoma [7]. There are also multiple ongoing trials (NCT03454919, NCT03242382, NCT01037790, and NCT02806648) investigating efficacy and safety of palbociclib in multiple malignancies with CDK4 overexpression (www.clinicaltrials.gov). Palbociclib was therefore started upon resistance to treatment with bevacizumab, irinotecan, and temozolomide, with the patient's consent. The remarkable response of CDK4-amplified CNS tumor to palbociclib-based multi-modality therapy as observed in the present case was not seen in another study attempting to match high grade glioma patients with targeted agents based on genomic sequencing results. In that study, seven out of 43 (16.3\%) cases carried CDK4 amplification, and palbociclib failed to elicit any response in a 65-year old patient following 2-months of treatment [11]. There are two possible explanations for this discrepancy: first of all, the 65-year old patient in that study had more advanced disease with a low Karnofsky score (KPS) at the time of palbociclib treatment; secondly, although palbociclib was able to prolong survival in mouse models of glioma, it has low blood-brain barrier (BBB) permeability as indicated by an unbound brain-to-plasma partition coefficient (Kp, uu) of 0.01 five minutes following intravenous administration in xenografts [12-14]. In our case, the patient underwent gamma knife radiosurgery before palbociclib treatment which might have improved the intake of palbociclib, given multiple lines of evidence showing the destruction of BBB after radiotherapy [15].

Administration of bevacizumab, irinotecan, and temozolomide after radiation was not effective for disease control in our case. It was not surprising since the addition of bevacizumab to temozolomide only had palliative effects on patients' outcomes, and the value of chemotherapy and bevacizumab in spinal cord tumors is still inconclusive [16]. Another anti-angiogenic agent apatinib, however, was effective in patients with refractory high-grade gliomas when administered alongside chemotherapeutic agents such as temozolomide and irinotecan $[8,17]$. This could have, in part, contributed to the exceptional response of the patient to the palbociclib-apatinib-temozolomide regimen despite multiple lines of previous treatment. The divergent effects of apatinib and bevacizumab could be explained by the fact that apatinib targets the intracellular domain of vascular endothelial growth factor receptor 2 (VEGFR-2) and hence induces tumor cell apoptosis by inhibiting autocrine VEGF signaling $[18,19]$. Moreover, apatinib could reverse ATP-binding cassette $(\mathrm{ABC})$ transportermediated multidrug resistance and enhance the efficacy of chemotherapy [20]. 
This case is of particular interest to us because it is the first case of spinal cord tumor ever reported to demonstrate an association between CDK4 amplification and response to palbociclib-based combination therapy even after multiple recurrences. The success with this case corroborates the notion that both comprehensive genomic profiling and a multi-modality treatment strategy are critical for personalized therapy of rare cancer types.

\section{Supplementary information}

Supplementary information accompanies this paper at https://doi.org/10. 1186/s12885-020-07061-3.

Additional file 1: Table S1. Markers examined using IHC. Table S2. A

list of the 381 genes included in the NGS panel

\section{Abbreviations}

ABC: ATP-binding cassette; Al: Aromatase inhibitor; BBB: Blood-brain barrier; CDK4: Cyclin dependent kinase 4; CNS: Central nervous system; CCND1: Cyclin D; CT: Computed tomography; FRS2: Fibroblast growth factor receptor substrate 2; GLI1: GLI family zinc finger 1; HR+: Hormone receptor positive.; H3F3A: H3.3 histone A; HIST1H3B: Histone cluster 1, H3b; IDH: Isocitrate dehydrogenase; KPS: Karnofsky score; MBC: Metastatic breast cancer; MDM2: MDM2 proto-oncogene; MRI: Magnetic resonance imaging; NCCN: National Comprehensive Cancer Network; NGS: Next-generation sequencing; PFS: Progression free survival; PTPN11: Protein tyrosine phosphatase non-receptor type 11; PET-CT: Positron emission tomographycomputed tomography; SCA: Spinal cord astrocytoma; VEGFR-2: Vascular endothelial growth factor receptor 2; WEE1: WEE1 G2 checkpoint kinase

\section{Acknowledgments}

The authors would like to thank Zhongsheng Kuang, Ph.D. from the first affiliated hospital of Guangzhou University of Chinese Medicine, for providing information on histologic results.

\section{Authors' contributions}

$L Z L$ designed and analyzed the data. $Y C$ drafted and revised the manuscript. JT L, L Y, YF F, HR C, XT Z, ST W, Y C made contributions to follow up the patient and acquisition of data. JT L, YF F, C G had wrote the original draft. JT L, C G had reviewed and edited the final version. JT L and $L$ $Y$ contributed equally. $L Z L$ and $Y C$ contributed equally. All authors read and approved the final manuscript.

\section{Funding}

The research reported in this publication was partially supported by the grand from Science and Technology Planning Project of Guangdong Province South China traditional Chinese medicine Collaborative Innovation Center, No.2014B090902002. The grant supported this study just financially and had no role in the design of the study and collection, analysis, and interpretation of data and in writing the manuscript.

\section{Availability of data and materials}

The datasets generated and analyzed during this study are not publicly available but are available from the corresponding author on reasonable request.

\section{Ethics approval and consent to participate}

This study conforms to the ethical guidelines for human research and the regulations of the Ethics Committee of the First Affiliated Hospital of Guangzhou University of Traditional Chinese Medicine. Ethical approval was waived. Written informed consent was obtained from the patient before NGS testing was performed.

\section{Consent for publication}

The patient and his family provided written informed consent for the publication of the present case report.

\section{Competing interests}

The authors declare that they have no competing interests.

\section{Author details}

${ }^{1}$ Oncology Center, the First Affiliated Hospital of Guangzhou University of Chinese Medicine, 16th Airport Road, Guangzhou 510405, Guangdong,

China. ${ }^{2}$ Guangzhou University of Chinese Medicine, 12th Airport Road, Guangzhou 510405, Guangdong, China. '3hanghai Institute of Cardiovascular Diseases, Zhongshan Hospital, Fudan University, 180 Fenglin Road, Shanghai 200032, China. ${ }^{4}$ Medical Affairs,3D Medicines Inc., Building 2, Block B, 158 XinJunhuan Street, Pujiang Hi-tech Park, MinHang District, Shanghai 201114, China.

Received: 24 August 2019 Accepted: 11 June 2020

Published online: 08 July 2020

\section{References}

1. Roonprapunt C, Houten JK. Spinal cord astrocytomas: presentation, management, and outcome. Neurosurg Clin N Am. 2006;17(1):29-36.

2. Duong LM, McCarthy BJ, McLendon RE, et al. Descriptive epidemiology of malignant and nonmalignant primary spinal cord, spinal meninges, and cauda equina tumors, United States, 2004-2007. Cancer. 2012; 118(17):4220-7.

3. Ostrom QT, Gittleman H, Xu J, et al. CBTRUS Statistical Report: Primary Brain and Other Central Nervous System Tumors Diagnosed in the United States in 2009-2013. Neuro Oncol. 2016. 18(suppl_5): v1-v75.

4. Teng YD, Abd-El-Barr M, Wang L, Hajiali H, Wu L, Zafonte RD. Spinal cord astrocytomas: progresses in experimental and clinical investigations for developing recovery neurobiology-based novel therapies. Exp Neurol. 2019; 311:135-47.

5. Hamilton E, Infante JR. Targeting CDK4/6 in patients with cancer. Cancer Treat Rev. 2016;45:129-38.

6. Schettini F, De Santo I, Rea CG, et al. CDK 4/6 inhibitors as single agent in advanced solid tumors. Front Oncol. 2018;8:608.

7. Dickson MA, Tap WD, Keohan ML, et al. Phase II trial of the CDK4 inhibitor PD0332991 in patients with advanced CDK4-amplified well-differentiated or dedifferentiated liposarcoma. J Clin Oncol. 2013:31(16):2024-8.

8. Wang L, Liang L, Yang T, et al. A pilot clinical study of apatinib plus irinotecan in patients with recurrent high-grade glioma: clinical trial/ experimental study. Medicine (Baltimore). 2017;96(49):e9053.

9. National Comprehensive Cancer Network Guidelines Steering, C. Central Nervous System Cancers (Version 1. 2019) Available from. National Comprehensive Cancer Network Guidelines Steering, C. Central Nervous System Cancers (Version 1. 2019).

10. Reifenberger G, Ichimura K, Reifenberger J, Elkahloun AG, Meltzer PS, Collins VP. Refined mapping of 12q13-q15 amplicons in human malignant gliomas suggests CDK4/SAS and MDM2 as independent amplification targets. Cancer Res. 1996:56(22):5141-5.

11. Blumenthal DT, Dvir A, Lossos A, et al. Clinical utility and treatment outcome of comprehensive genomic profiling in high grade glioma patients. J Neuro-Oncol. 2016;130(1):211-9.

12. Michaud K, Solomon DA, Oermann E, et al. Pharmacologic inhibition of cyclin-dependent kinases 4 and 6 arrests the growth of glioblastoma multiforme intracranial xenografts. Cancer Res. 2010;70(8):3228-38.

13. Barton KL, Misuraca K, Cordero F, et al. PD-0332991, a CDK4/6 inhibitor, significantly prolongs survival in a genetically engineered mouse model of brainstem glioma. PLoS One. 2013;8(10):e77639.

14. Raub TJ, Wishart GN, Kulanthaivel P, et al. Brain exposure of two selective dual CDK4 and CDK6 inhibitors and the antitumor activity of CDK4 and CDK6 inhibition in combination with Temozolomide in an intracranial Glioblastoma Xenograft. Drug Metab Dispos. 2015;43(9):1360-71.

15. Appelboom G, Detappe A, LoPresti M, et al. Stereotactic modulation of blood-brain barrier permeability to enhance drug delivery. Neuro-Oncology. 2016;18(12):1601-9.

16. van den Bent MJ, Klein M, Smits M, et al. Bevacizumab and temozolomide in patients with first recurrence of WHO grade II and III glioma, without 1p/ 19q co-deletion (TAVAREC): a randomised controlled phase 2 EORTC trial. Lancet Oncol. 2018;19(9):1170-9.

17. Wang C, Jiang M, Hou H, Lin Q, Yan Z, Zhang X. Apatinib suppresses cell growth and metastasis and promotes antitumor activity of temozolomide in glioma. Oncol Lett. 2018;16(5):5607-14. 
18. Peng $H$, Zhang $Q$, Li J, et al. Apatinib inhibits VEGF signaling and promotes apoptosis in intrahepatic cholangiocarcinoma. Oncotarget. 2016;7(13): 17220-9.

19. Peng $\mathrm{S}$, Zhang $\mathrm{Y}$, Peng $\mathrm{H}$, et al. Intracellular autocrine VEGF signaling promotes EBDC cell proliferation, which can be inhibited by Apatinib. Cancer Lett. 2016;373(2):193-202.

20. Mi YJ, Liang YJ, Huang HB, et al. Apatinib (YN968D1) reverses multidrug resistance by inhibiting the efflux function of multiple ATP-binding cassette transporters. Cancer Res. 2010;70(20):7981-91.

\section{Publisher's Note}

Springer Nature remains neutral with regard to jurisdictional claims in published maps and institutional affiliations.

Ready to submit your research? Choose BMC and benefit from:

- fast, convenient online submission

- thorough peer review by experienced researchers in your field

- rapid publication on acceptance

- support for research data, including large and complex data types

- gold Open Access which fosters wider collaboration and increased citations

- maximum visibility for your research: over $100 \mathrm{M}$ website views per year

At BMC, research is always in progress.

Learn more biomedcentral.com/submissions 